\title{
Fast Converging Decentralized WSRMax for MIMO IBC with Low Computational Complexity
}

\author{
Jarkko Kaleva, Antti Tölli and Markku Juntti \\ Centre for Wireless Communications, University of Oulu \\ University of Oulu, P.O. Box 4500, 90014, Finland \\ firstname. lastnamedoulu.fi
}

\begin{abstract}
Iteratively decentralized weighted sum rate maximization (WSRMax) is proposed for multiple-input multipleoutput (MIMO) interfering broadcast channel. Particular emphasis is given for improved rate of convergence for the WSRMax utility. Successive convex approximation is applied to provide an algorithm with fast rate of convergence and low computational complexity per iteration while sustaining the monotonic improvement of the objective. This method has particularly convenient structure for decentralized processing allowing alternating receive and transmit beamformer updates. This structure complies with recently proposed low overhead pilot aided beamformer signaling frameworks. The computational complexity and signaling overhead of the scheme are equivalent with the well-established weighted mean squared error minimization (WMMSE) approach. The proposed method is shown, by numerical examples, to improve the rate of convergence with respect to the WMMSE and semidefinite program (SDP) relaxation methods.
\end{abstract}

\section{INTRODUCTION}

The capacity requirements of modern wireless systems are becoming increasingly demanding. In an effort to increase the throughput, the cell sizes are decreased and inter-cell cooperation is more important than ever before. The fifth generation $(5 \mathrm{G})$ systems are heterogeneous in the sense that they support varying architectures simultaneously in the shared spatial and frequency domain. This imposes new challenges for interference coordination. In the spatial domain, multiuser multiple-input multiple-output (MIMO) systems provide potentially significant performance improvements, by allowing multiple simultaneous and independent data streams on the shared spectrum. However, the inter-cell beam coordination for the simultaneous transmissions, particularly, with limited information exchange and fast changing interference conditions is one of the major obstacles preventing wide adaptation of the advanced MIMO techniques.

We consider decentralized weighted sum rate maximization (WSRMax) in MIMO interfering broadcast channel (IBC). The proposed approach imposes two successive convex approximation (SCA) stages that transform the initially non-convex and NP-hard problem into computationally tractable convex subproblems [1]. This algorithms can be shown to posses a convenient structure for decentralized processing by allowing iterative transmit/receive beamformer updates. For instance, in time division duplex (TDD) the transceiver processing is temporally separated, and, thus, benefits from alternating beamformer updates. Alternating transceiver processing has been well studied [2], [3] and we have shown that the proposed scheme can be readily applied to available pilot based TDD signaling frameworks proposed, e.g., in [3], [4]. Many practical issues involved with the multi-user beamforming, such as, signaling overhead [3] and computational complexity [2] have gained considerably more attention than the convergence properties. The rate of convergence also contributes to the overall computational complexity, and, as such, is an important property for any practically realizable algorithm. We will show that the proposed design provides monotonic convergence with respect to the WSRMax objective. The computational complexity and signaling overhead of the scheme are equivalent with the well-established weighted minimum MSE (WMMSE) approach [2], [5]. The proposed method is shown, by numerical examples, to improve the rate of convergence with respect to the WMMSE [2], [5] and semidefinite program (SDP) relaxation methods [6].

In [7], [8], the WSRMax objective was reformulated as the sum of difference of logarithms that is a difference of convex functions program (DCP). By iteratively approximating the non-convex terms of the objective function, the algorithm converges and a low complexity solution is achieved. An alternative approximation procedure is given in [9], where each approximated step was given as a geometric program (GP) [10]. Similarly, iterative WSRMax by alternating beamforming and power loading was proposed in [11], [12]. In [13] a fast converging transmit beamformer design was proposed for multiple-input single-output (MISO) channel. It was also shown in [13] that the WMMSE methods have slow rate of convergence in MISO systems, particularly, on high signal-tonoise ratio $(\mathrm{SNR})$ regime.

Fast converging IBC (FIBC) WSRMax based on the WMMSE criterion has been widely adapted for varying system models [2], [3], [5], [14]. In the WMMSE approach, the objective function of the non-convex mean-squared error (MSE) formulation is iteratively approximated using successive firstorder approximation. This problem is convex when either the transmitters or receivers are fixed. The practical signaling issues were further studied in [3].

\section{SySTEM MODEL}

We consider a $B$-base station (BS) and $K$-user MIMO IBC with $N_{\mathrm{T}}$ transmit and $N_{\mathrm{R}}$ receive antennas. Each user $k=$ $1, \ldots, K$ is associated to a single BS denoted correspondingly as $b_{k}$ and the set of user indices associated to BS $b$ is denoted by $\mathcal{U}_{b}$ with $K_{b}=\left|\mathcal{U}_{b}\right|$. The channel matrix between $\mathrm{BS} b$ 
and user equipment (UE) $k=1, \ldots, K$ is denoted by $\mathbf{H}_{b, k} \in$ $\mathbb{C}^{N_{\mathrm{R}} \times N_{\mathrm{T}}}$. The maximum number of spatial data streams for each user is bounded by the available single-cell degrees of freedom (DoF), i.e., $N_{\min }=\min \left(N_{\mathrm{T}}, K_{b} N_{\mathrm{R}}\right)$. The received signal at user terminal $k=1, \ldots, K$ can be written as

$$
\mathbf{y}_{k}=\sum_{i=1}^{K} \mathbf{H}_{b_{i}, k} \mathbf{M}_{i} \mathbf{d}_{i}+\mathbf{n}_{k}
$$

where the normalized data symbols $\mathbf{d}_{k} \in \mathbb{C}^{N_{\text {min }}}$ are given as $\mathbb{E}\left[\left\|\mathbf{d}_{k}\right\|^{2}\right]=1$. The receiver noise is assumed to be circularly symmetric zero mean $\mathbf{n}_{k} \sim \mathcal{C N}\left(\mathbf{0}, \mathbf{R}_{k}\right)$, where $\mathbf{R}_{k}=\mathbb{E}\left[\mathbf{n}_{k} \mathbf{n}_{k}^{\mathrm{H}}\right]=\sigma_{k}^{2} \mathbf{I}$. The transmit beamformer matrix for user $k=1, \ldots, K$ is given as $\mathbf{M}_{k} \in \mathbb{C}^{N_{\mathrm{T}} \times N_{\min }}$. The estimated signal at the receiver $k$ is then given by $\hat{\mathbf{d}}_{k}=\mathbf{U}_{k}^{\mathrm{H}} \mathbf{y}_{k}$, where $\mathrm{U}_{k} \in \mathbb{C}^{N_{\mathrm{R}} \times N_{\text {min }}}$ denotes the linear receive beamformer matrix. Now, the error covariance of the received signal becomes

$$
\begin{aligned}
& \mathbf{E}_{k}=\mathbb{E}\left[\left(\hat{\mathbf{d}}_{k}-\mathbf{d}_{k}\right)\left(\hat{\mathbf{d}}_{k}-\mathbf{d}_{k}\right)^{\mathrm{H}}\right]=\mathbf{I}-\mathbf{U}_{k}^{\mathrm{H}} \mathbf{H}_{b_{k}, k} \mathbf{M}_{k}- \\
& \mathbf{M}_{k}^{\mathrm{H}} \mathbf{H}_{b_{k}, k}^{\mathrm{H}} \mathbf{U}_{k}+\sum_{i=1}^{K} \mathbf{U}_{k}^{\mathrm{H}} \mathbf{H}_{b_{i}, k} \mathbf{M}_{i} \mathbf{M}_{i}^{\mathrm{H}} \mathbf{H}_{b_{i}, k}^{\mathrm{H}} \mathbf{U}_{k}+\mathbf{U}_{k}^{\mathrm{H}} \mathbf{R}_{k} \mathbf{U}_{k} .
\end{aligned}
$$

The diagonal elements of $\mathbf{E}_{k}$ give the MSE of the estimated symbols.

\section{PROBLEM FORMULATION}

We consider WSRMax in IBC, where the BSs are subject to sum transmit power constraints $\sum_{k \in \mathcal{U}_{b}} \operatorname{Tr}\left[\mathbf{M}_{k}^{\mathrm{H}} \mathbf{M}_{k}\right] \leq P, b=$ $1, \ldots, B$, while the proposed method can be straightforwardly extended to other power constraints as well. This problem can be formulated as

$$
\begin{aligned}
\max _{\mathbf{M}_{k}} & \sum_{k=1}^{K} \mu_{k} \log \operatorname{det}\left(\mathbf{I}+\mathbf{M}_{k}^{\mathrm{H}} \mathbf{H}_{b_{k}, k}^{\mathrm{H}} \mathbf{Q}_{k}^{-1} \mathbf{H}_{b_{k}, k} \mathbf{M}_{k}\right) \\
\text { s. t. } & \sum_{k \in \mathcal{U}_{b}} \operatorname{Tr} \mathbf{M}_{k} \mathbf{M}_{k}^{\mathrm{H}} \leq P, b=1, \ldots, B,
\end{aligned}
$$

where the priority weights for each users are denoted by $\mu_{k}, k=1, \ldots, K$. The interference-plus-noise covariance matrix $\mathbf{Q}_{k}$ at UE $k$ is

$$
\mathbf{Q}_{k}=\sum_{i=1, i \neq k}^{K} \mathbf{H}_{b_{i}, k} \mathbf{M}_{i} \mathbf{M}_{i}^{\mathrm{H}} \mathbf{H}_{b_{i}, k}^{\mathrm{H}}+\mathbf{R}_{k} .
$$

Note that formulation in (3) does not explicitly include the receive beamformer notation $\mathbf{U}_{k}$. This formulation implicitly assumes that the rate optimal linear minimum MSE (MMSE) receivers are applied. The MMSE receiver for UE $k$ is

$$
\left.\mathbf{U}_{k}=\sum_{i=1}^{K} \mathbf{H}_{b_{i}, k} \mathbf{M}_{i} \mathbf{M}_{i}^{\mathrm{H}} \mathbf{H}_{b_{i}, k}^{\mathrm{H}}+\mathbf{R}_{k}\right)^{-1} \mathbf{H}_{b_{k}, k} \mathbf{M}_{k} .
$$

The terms in (3) can be rewritten as a difference of log det functions

$$
\begin{aligned}
& \log \operatorname{det}\left(\mathbf{Q}_{k} \mathbf{Q}_{k}^{-1}+\mathbf{H}_{b_{k}, k} \mathbf{M}_{k} \mathbf{M}_{k}^{\mathrm{H}} \mathbf{H}_{b_{k}, k}^{\mathrm{H}} \mathbf{Q}_{k}^{-1}=\right. \\
& \left.\log \operatorname{det} \mathbf{Q}_{k}+\mathbf{H}_{b_{k}, k} \mathbf{M}_{k} \mathbf{M}_{k}^{\mathrm{H}} \mathbf{H}_{b_{k}, k}^{\mathrm{H}}\right)-\log \operatorname{det}\left(\mathbf{Q}_{k}\right) .
\end{aligned}
$$

To further simplify the notation, we define

$$
\mathbf{C}_{k}=\mathbf{Q}_{k}+\mathbf{H}_{b_{k}, k} \mathbf{M}_{k} \mathbf{M}_{k}^{\mathrm{H}} \mathbf{H}_{b_{k}, k}^{\mathrm{H}}
$$

and have the objective as

$$
\sum_{k=1}^{K} \mu_{k}\left(\log \operatorname{det}\left(\mathbf{C}_{k}\right)-\log \operatorname{det}\left(\mathbf{Q}_{k}\right)\right),
$$

where $\mathbf{C}_{k}$ and $\mathbf{Q}_{k}$ are both quadratic functions of $\mathbf{M}_{i}, i=$ $1, \ldots, K$. This form is convenient for convex approximation as the objective is a composite of convex and concave functions. In the sequel, as the SCA algorithm is developed to iteratively approximate the WSRMax objective. We begin by briefly deriving the outline of the WMMSE methods, which is used as a point of comparison for the proposed designs.

\section{WMMSE REFERENCE DESIGN}

When the optimal receivers (5) are applied to (2), there exists the following well-known inverse relation

$$
\mathbf{E}_{k}^{-1}=\mathbf{I}+\mathbf{H}_{b_{k}, k} \mathbf{M}_{k} \mathbf{Q}_{k}^{-1} \mathbf{M}_{k}^{\mathrm{H}} \mathbf{H}_{b_{k}, k}^{\mathrm{H}} .
$$

Note that the diagonal elements in (9) represent the stream specific signal-to-interference-plus-noise ratio (SINR) values.

Using the relation in (9), the WSRMax problem (3) can be written as the corresponding MSE minimization problem

$$
\begin{aligned}
\min _{\mathbf{U}_{k}, \mathbf{M}_{k}} & \sum_{k=1}^{K} \mu_{k} \log \operatorname{det}\left(\mathbf{E}_{k}\right) \\
\text { s. t. } & \sum_{k \in \mathcal{U}_{b}} \operatorname{Tr} \mathbf{M}_{k} \mathbf{M}_{k}^{\mathrm{H}} \leq P, b=1, \ldots, B,
\end{aligned}
$$

where $\mathbf{E}_{k}$ is from (2). The optimal receive beamformers for (10) can be straightforwardly derived from the KarushKuhn-Tucker (KKT) conditions and are the same MMSE receivers as given in (5). Since (5) is the optimal solution for (10), we can assure that (9) holds in any optimum. Thus, we can show that problems (3) and (10) are equivalent in terms of the optimal solution [5].

The log det objective function can be linearly approximated by the conventional first-order Taylor series expansion. In each iteration, the corresponding point of approximation is given by the previous iteration MSE matrices $\left\{\overline{\mathbf{E}}_{k}\right\}_{k=1}^{K}$. The approximated objective of (10) is given as

$$
\sum_{k=1}^{K}\left(\mu_{k} \log \operatorname{det} \overline{\mathbf{E}}_{k}+\mu_{k} \operatorname{Tr} \overline{\mathbf{E}}_{k}^{-1}\left(\mathbf{E}_{k}-\overline{\mathbf{E}}_{k}\right)\right.
$$

Herein, we use the notation $\overline{\mathbf{E}}$ to denote the fixed value of variable $\mathbf{E}$ from the previous iteration. Using (11), the corresponding subproblem can be formulated as

$$
\begin{aligned}
\underset{\mathbf{U}_{k}, \mathbf{M}_{k}}{\min } & \sum_{k=1}^{K} \mu_{k} \operatorname{Tr}\left(\mathbf{W}_{k} \mathbf{E}_{k}\right) \\
\text { s. t. } & \sum_{k \in \mathcal{U}_{b}} \operatorname{Tr} \mathbf{M}_{k} \mathbf{M}_{k}^{\mathrm{H}} \leq P, b=1, \ldots, B,
\end{aligned}
$$

where the linearization coefficients are given as

$$
\mathbf{W}_{k}=\overline{\mathbf{E}}_{k}^{-1}, k=1, \ldots, K .
$$

The constant terms are neglected in (12) as they have no impact on the solution [15]. 
The WMMSE precoder can be solved via alternating transceiver updates in [2], [3], [5]. For fixed receive beamformers, the optimal transmit beamformers can be obtained as [2]

$$
\mathbf{M}_{k}=\mathbf{T}_{b_{k}}^{-1} \mathbf{H}_{b_{k}, k}^{\mathrm{H}} \mathbf{U}_{k} \mathbf{W}_{k}, k=1, \ldots, K,
$$

where the weighted transmit covariance matrix is given as

$$
\mathbf{T}_{b}=\sum_{i=1}^{K} \mathbf{H}_{b, i}^{\mathrm{H}} \mathbf{U}_{i} \mathbf{W}_{i} \mathbf{U}_{i}^{\mathrm{H}} \mathbf{H}_{b, i}+\nu_{b} \mathbf{I}
$$

and $\nu_{b}$ denotes the Lagrange multiplier for the sum power constraint. The optimal value for each $\nu_{b}, b=1, \ldots, B$ is found by bisection search to satisfy the power constraints [2].

\section{Proposed FIBC Design}

In this section, we propose a fast converging MIMO beamformer design for the proposed system model. This approach provides an algorithm similar to the WMMSE design with comparable computational complexity and signaling structure for decentralized processing. However, in Section VI, we show by numerical evaluation that the proposed design has significantly improved convergence properties.

Similar to (11), we begin by linearly approximating the $\log$ det (convex) functions in the objective in terms of $\mathbf{C}_{k}$ and $\mathbf{Q}_{k}$ for all $k=1, \ldots, K$. This results in

$$
\begin{aligned}
\max _{\mathbf{M}_{k}} & \sum_{k=1}^{K} \mu_{k} \operatorname{Tr} \overline{\mathbf{C}}_{k}^{-1} \mathbf{C}_{k}-\operatorname{Tr} \overline{\mathbf{Q}}_{k}^{-1} \mathbf{Q}_{k} \\
\text { s. t. } & \sum_{k \in \mathcal{U}_{b}}^{K} \operatorname{Tr} \mathbf{M}_{k} \mathbf{M}_{k}^{\mathrm{H}} \leq P, b=1, \ldots, B .
\end{aligned}
$$

The linear approximation of a convex function provides a global lower bound for the maximization objective. By regrouping the objective terms, (16) can be rewritten as

$$
\begin{aligned}
\min _{\mathbf{M}_{k}} & \sum_{k=1}^{K} \mu_{k} \operatorname{Tr} \quad \overline{\mathbf{Q}}_{k}^{-1}-\overline{\mathbf{C}}_{k}^{-1} \mathbf{Q}_{k}- \\
\text { s. t. } & \sum_{k=1}^{K} \mu_{k} \operatorname{Tr} \overline{\mathbf{C}}_{k}^{-1} \mathbf{H}_{b_{k}, k} \mathbf{M}_{k} \mathbf{M}_{k}^{\mathrm{H}} \mathbf{H}_{b_{k}, k}^{\mathrm{H}} \mathbf{M}_{k} \mathbf{M}_{k}^{\mathrm{H}} \leq P, b=1, \ldots, B .
\end{aligned}
$$

Note that this problem is still non-convex due to the latter part of the objective.

We further simplify the notation by applying the matrix inversion lemma to $\overline{\mathbf{C}}_{k}^{-1}-\overline{\mathbf{Q}}_{k}^{-1}$ as

$$
\begin{aligned}
& \overline{\mathbf{C}}_{k}^{-1}-\overline{\mathbf{Q}}_{k}^{-1}=\left(\overline{\mathbf{Q}}_{k}+\mathbf{H}_{b_{k}, k}^{\mathrm{H}} \overline{\mathbf{M}}_{k}^{\mathrm{H}} \overline{\mathbf{M}}_{k} \mathbf{H}_{b_{k}, k}{ }^{-1}-\overline{\mathbf{Q}}_{k}^{-1}=\right. \\
& \overline{\mathbf{M}}_{k}^{\mathrm{H}} \mathbf{H}_{b_{k}, k}^{\mathrm{H}} \overline{\mathbf{Q}}_{k}^{-1} \mathbf{I}+\mathbf{H}_{b_{k}, k} \overline{\mathbf{M}}_{k} \overline{\mathbf{Q}}_{k}^{-1} \overline{\mathbf{M}}_{k}^{\mathrm{H}} \mathbf{H}_{b_{k}, k}^{\mathrm{H}} \overline{\mathbf{Q}}_{k}^{-1} \mathbf{H}_{b_{k}, k} \overline{\mathbf{M}}_{k} \\
& =\overline{\mathbf{U}}_{k} \overline{\mathbf{W}}_{k} \overline{\mathbf{U}}_{k}^{\mathrm{H}},
\end{aligned}
$$

where $\mathbf{U}_{k}$ is the MMSE receiver (5) and $\mathbf{W}_{k}$ is the MSE weight matrix (12). It can be directly observed from (18) that $\overline{\mathbf{C}}_{k}^{-1}-\overline{\mathbf{Q}}_{k}^{-1}$ is always positive semidefinite (PSD). By this notion, the only remaining non-convex (concave) terms in the objective of (17) are $-\operatorname{Tr} \quad \overline{\mathbf{C}}_{k}^{-1} \mathbf{H}_{b_{k}, k} \mathbf{M}_{k} \mathbf{M}_{k}^{\mathrm{H}} \mathbf{H}_{b_{k}, k}^{\mathrm{H}}, k=$
$1, \ldots, K$. We perform a second linear approximation to transform these terms into a linear form as

$$
\begin{aligned}
& \mathcal{L}\left(\overline{\mathbf{C}}_{k}^{-1} \mathbf{H}_{b_{k}, k} \mathbf{M}_{k} \mathbf{M}_{k}^{\mathrm{H}} \mathbf{H}_{b_{k}, k}^{\mathrm{H}}=\operatorname{Tr} \overline{\mathbf{C}}_{k}^{-1} \mathbf{H}_{b_{k}, k} \overline{\mathbf{M}}_{k} \overline{\mathbf{M}}_{k}^{\mathrm{H}} \mathbf{H}_{b_{k}, k}^{\mathrm{H}}\right. \\
& +\operatorname{Tr}\left(\overline{\mathbf{C}}_{k}^{-1} \mathbf{H}_{b_{k}, k} \mathbf{M}_{k}-\overline{\mathbf{M}}_{k} \overline{\mathbf{M}}_{k}^{\mathrm{H}} \mathbf{H}_{b_{k}, k}^{\mathrm{H}}\right. \\
& +\operatorname{Tr} \overline{\mathbf{C}}_{k}^{-1} \mathbf{H}_{b_{k}, k} \overline{\mathbf{M}}_{k} \mathbf{M}_{k}-\overline{\mathbf{M}}_{k}{ }^{\mathrm{H}} \mathbf{H}_{b_{k}, k}^{\mathrm{H}}= \\
& \operatorname{Tr}\left(\overline{\mathbf{U}}_{k}^{\mathrm{H}} \mathbf{H}_{b_{k}, k} \mathbf{M}_{k}+\operatorname{Tr} \mathbf{M}_{k}^{\mathrm{H}} \mathbf{H}_{b_{k}, k}^{\mathrm{H}} \overline{\mathbf{U}}_{k}-\right. \\
& \operatorname{Tr} \overline{\mathbf{C}}_{k}^{-1} \mathbf{H}_{b_{k}, k} \overline{\mathbf{M}}_{k} \overline{\mathbf{M}}_{k}^{\mathrm{H}} \mathbf{H}_{b_{k}, k}^{\mathrm{H}} .
\end{aligned}
$$

Note that (17) is a minimization problem for which (19) provides a globe upper bound. Finally, using (19), we can formulate the convex subproblems as

$$
\begin{aligned}
\min _{\mathbf{M}_{k}} & \sum_{k=1}^{K}\left(\operatorname{Tr} \overline{\mathbf{W}}_{k} \mathbf{Q}_{k}-\operatorname{Tr} \overline{\mathbf{U}}_{k}^{\mathrm{H}} \mathbf{H}_{b_{k}, k} \mathbf{M}_{k}-\right. \\
\text { s. t. } & \operatorname{Tr}\left(\mathbf{M}_{k}^{\mathrm{H}} \mathbf{H}_{b_{k}, k}^{\mathrm{H}} \overline{\mathbf{U}}_{k}\right. \\
& \sum_{k \in \mathcal{U}_{b}} \operatorname{Tr} \mathbf{M}_{k} \mathbf{M}_{k}^{\mathrm{H}} \leq P, b=1, \ldots, B .
\end{aligned}
$$

Note that the constant terms have been neglected from the objective, as they have no impact on the optimal solution.

Now, by taking the first order optimality conditions of (20), the transmit beamformer can be solved in a closed form as

$$
\mathbf{M}_{k}=\mathbf{T}_{b_{k}}^{-1} \mathbf{H}_{b_{k}, k}^{\mathrm{H}} \overline{\mathbf{U}}_{k}, k=1, \ldots, K,
$$

where the transmit covariance matrix is given as

$$
\mathbf{T}_{b}=\sum_{i=1, i \neq k}^{K} \mathbf{H}_{b, i}^{\mathrm{H}} \overline{\mathbf{U}}_{i} \overline{\mathbf{W}}_{i} \overline{\mathbf{U}}_{i}^{\mathrm{H}} \mathbf{H}_{b, i}+\nu_{b} \mathbf{I} .
$$

The optimal transmit beamformers can be determined by bisection search over $\nu_{b}$ to satisfy the transmit power constraints $\sum_{k \in \mathcal{U}_{b}} \operatorname{Tr}\left[\mathbf{M}_{k}^{\mathrm{H}} \mathbf{M}_{k}\right] \leq P, b=1, \ldots, B$. The algorithm is summarized by Algorithm 1 .

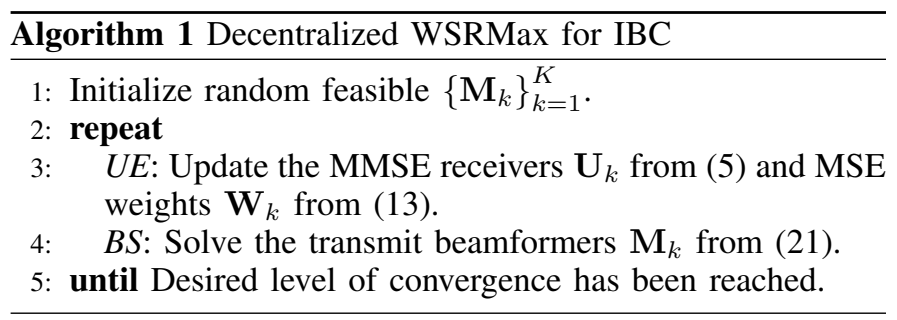

The proposed solution (21) is very similar to (14) though with some key differences. Note that in (22) does not contain the covariance of the weighted intended signal $\mathbf{H}_{b, k}^{\mathrm{H}} \mathbf{U}_{k} \mathbf{W}_{k} \mathbf{U}_{k}^{\mathrm{H}} \mathbf{H}_{b, k}$ for the corresponding UE $k$. Also the matched filter (MF) part of the solution does not contain the corresponding weight $\mathbf{H}_{k}^{\mathrm{H}} \mathbf{U}_{k}$. These differences provide the improved convergence properties. The intuition is that the algorithm does not rely as heavily on the previous iteration fixed linearization coefficients as the WMMSE solution does, which improves the rate of convergences.

\section{Objective function convergence}

We can show that each iteration provides a monotonic improvement in the objective function. First, the problem is 
formulated in an equivalent form as a DCP

$$
\begin{aligned}
\underset{\mathbf{M}_{k}, \mathbf{A}_{k}, \mathbf{B}_{k}}{\min } & \sum_{k=1}^{K} \mu_{k}\left(\log \operatorname{det}\left(\mathbf{A}_{k}\right)-\log \operatorname{det}\left(\mathbf{B}_{k}\right)\right) \\
\text { s. t. } & \mathbf{Q}_{k}+\mathbf{H}_{b_{k}, k} \mathbf{M}_{k} \mathbf{M}_{k}^{\mathrm{H}} \mathbf{H}_{b_{k}, k}^{\mathrm{H}} \preceq \mathbf{A}_{k}, k=1, \ldots, K, \\
& \mathbf{Q}_{k} \succeq \mathbf{B}_{k}, k=1, \ldots, K, \\
& \sum_{k \in \mathcal{U}_{b}} \operatorname{Tr} \mathbf{M}_{k} \mathbf{M}_{k}^{\mathrm{H}} \leq P, b=1, \ldots, B .
\end{aligned}
$$

In this form, all terms in the problem are either convex or concave. Since the linear approximation in both steps (16) and (19) are done with respect to convex/concave functions they provide global under/overestimators that are tight in the point of approximation [15]. Thus, each subproblem solution has to improve the objective value of the original problem and leads to monotonic improvement. It was shown in [16] that either the solution for the SCA subproblem is a solution of the original problem or the objective is monotonically improved. Since the objective function is bounded by the power constraints, the objective of (16) converges. The beamformer convergence analysis is out of the scope of this paper (for more details see, e.g., [17] for convergence analysis with similar transceiver design).

\section{Decentralized processing and signaling}

It turns out that the signaling requirements for decentralized processing are effectively the same as those for the WMMSE method and the proposed scheme. Note that in (21) and (22), the beamformer solution for $\mathbf{M}_{k}$ does not depend on $\mathbf{M}_{i}, i \neq k$ and is, as such, decoupled. This enables the iteratively decentralized processing. To construct (22) for UE $k$, BS $b_{k}$ needs to know the effective downlink channels $\mathbf{H}_{b, i}^{\mathrm{H}} \mathbf{U}_{i} \mathbf{W}_{i}^{\frac{1}{2}}, i \neq k$. Additionally, for the intended user, the non-weighted channel $\left(\mathbf{H}_{b, k}^{\mathrm{H}} \mathbf{U}_{k}\right)$ needs to be known. The signaling schemes for pilot based effective channel state information (CSI) exchange have been studied, e.g., in [3].

\section{NUMERICAL EXAMPLES}

The numerical performance evaluation is carried out with i.i.d. Rayleigh fading channels. The SNR at each user terminal $k=1, \ldots, K$ is defined as $P / \sigma_{k}^{2}$. For the sake of simplicity, the priority weights for all UEs are equal $\left(\mu_{k}=1 \forall k\right)$. The performance is compared to that of the WMMSE method as proposed in [2], [5]. The log det reference uses approximation method from [6], where SDP relaxation is used for the beamformer matrices and only a single level linear approximation is required. Note that this method has higher per-iteration computational complexity as each subproblem is a SDP. The proposed design is denoted by LinearFIBC in the figures.

Fig. 1 shows the achievable rate on limited number of beamformer iterations and varying SNR in a four cell system with $K_{b}=4$ UEs per cell. There are $N_{\mathrm{T}}=4$ transmit and $N_{\mathrm{R}}=4$ receive antennas. It is evident that, as the SNR increases, the proposed scheme provides significant gain over the WMMSE method. For both schemes, it takes a few iterations for the randomly initialized beamformer to adapt, which can be seen by comparable performance for 3 beamformer iterations over the whole SNR range.

The convergence behavior, for fixed a SNR point, is demonstrated in Fig. 2 for two system configurations. In both

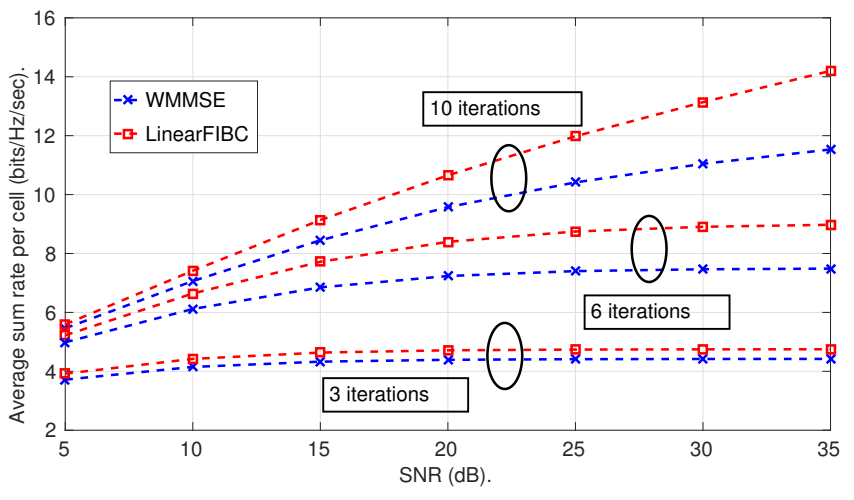

Fig. 1. Achievable rate with limited number of beamformer iterations.

cases, the SNR is fixed to $30 \mathrm{~dB}$. With $B=2$ and $K_{b}=8$, there are more users in each cell than there are available DoF and implicit user selection is performed by the algorithms. Due to the implicit user selection the log det method achieves high initial convergence rate, but slows down as the user selection settles. After approximately the $7^{\text {th }}$ iteration, the proposed design out performs the reference algorithms. In the more complex $B=4$ cell system, the proposed design provides the fastest rate of convergence from the second iteration.

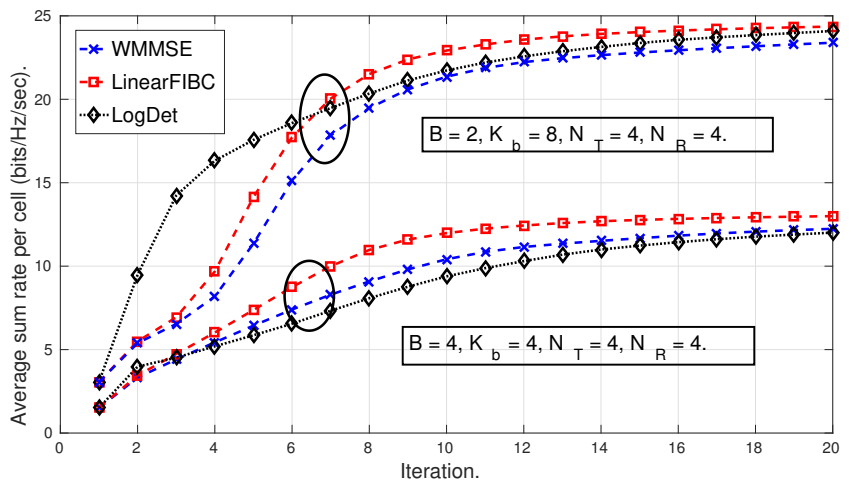

Fig. 2. Average rate of convergence for the WSRMax.

\section{CONCLUSIONS}

We proposed an iteratively decentralized MIMO beamformer design for IBC. Convergence analysis and basic signaling characteristics were also provided. The computational complexity and signaling overhead were shown to be comparable to well-known low complexity decentralized WMMSE method. The proposed method was shown to provide improved rate of convergence with respect to the WMMSE method. It was also shown to out perform centralized high complexity SDP relaxation method.

\section{REFERENCES}

[1] Z. Luo and S. Zhang, "Dynamic spectrum management: Complexity and duality," IEEE J. Select. Areas Commun., vol. 2, no. 1, pp. 57-73, Feb. 2008.

[2] Q. Shi, M. Razaviyayn, Z.-Q. Luo, and C. He, "An iteratively weighted MMSE approach to distributed sum-utility maximization for a MIMO interfering broadcast channel," IEEE Trans. Signal Processing, vol. 59, no. 9, pp. 4331-4340, Sep. 2011. 
[3] P. Komulainen, A. Tölli, and M. Juntti, "Effective CSI Signaling and Decentralized Beam Coordination in TDD Multi-Cell MIMO Systems," IEEE Trans. Signal Processing, vol. 61, no. 9, pp. 2204-2218, 2013.

[4] C. Shi, R. Berry, and M. Honig, "Bi-directional training for adaptive beamforming and power control in interference networks," IEEE Trans. Signal Processing, vol. 62, no. 3, pp. 607-618, Feb. 2014.

[5] S. S. Christensen, R. Agarwal, E. Carvalho, and J. Cioffi, "Weighted sum-rate maximization using weighted MMSE for MIMO-BC beamforming design," IEEE Trans. Wireless Commun., vol. 7, no. 12, pp. 4792-4799, Dec. 2008.

[6] M. Razaviyayn, M. Sanjabi, and Z. Q. Luo, "Linear transceiver design for interference alignment: Complexity and computation," IEEE Trans. Inform. Theory, vol. 58, no. 5, pp. 2896-2910, May 2012.

[7] P. Tsiaflakis, M. Diehl, and M. Moonen, "Distributed spectrum management algorithms for multiuser DSL networks," IEEE Trans. Signal Processing, vol. 56, no. 10, pp. 4825-4843, Oct. 2008.

[8] N. Vucic, S. Shi, and M. Schubert, "DC programming approach for resource allocation in wireless networks," Avignon, France, Jun. 42010 , pp. 360-366.

[9] J. Papandriopoulos and J. S. Evans, "SCALE: A low-complexity distributed protocol for spectrum balancing in multiuser DSL networks," IEEE Trans. Inform. Theory, vol. 55, no. 8, pp. 3711-3724, Aug. 2009.

[10] M. Chiang, C. W. Tan, D. P. Palomar, D. O’Neill, and D. Julian, "Power control by geometric programming," IEEE Trans. Wireless Commun., vol. 6, no. 7, pp. 2640-2651, Jul. 2007.
[11] A. Tölli, M. Codreanu, and M. Juntti, "Cooperative MIMO-OFDM cellular system with soft handover between distributed base station antennas," IEEE Trans. Wireless Commun., vol. 7, no. 4, pp. 14281440, Apr. 2008.

[12] S. Shi, M. Schubert, and H. Boche, "Per-antenna power constrained rate optimization for multiuser MIMO systems," in Smart Antennas, 2008. WSA 2008. International ITG Workshop on, Darmstadt, Germany, Feb. 2008, pp. 270-277.

[13] L. Tran, M. Hanif, A. Tölli, and M. Juntti, "Fast converging algorithm for weighted sum rate maximization in multicell MISO downlink," IEEE Signal Processing Lett., vol. 19, no. 12, pp. 872-875, Dec. 2012.

[14] D. Nguyen and T. Le-Ngoc, "Sum-rate maximization in the multicell MIMO multiple-access channel with interference coordination," IEEE Trans. Wireless Commun., vol. 13, no. 1, pp. 36-48, Jan. 2014

[15] S. Boyd and L. Vandenberghe, Convex Optimization. Cambridge, UK: Cambridge University Press, 2004.

[16] B. R. Marks and G. P. Wright, "A general inner approximation algorithm for nonconvex mathematical programs," Journal of the Operations Research Society of America, vol. 26, no. 4, pp. 681-683, Jul.-Aug. 1978.

[17] J. Kaleva, A. Tölli, and M. Juntti, "Decentralized sum rate maximization with QoS constraints for interfering broadcast channel via successive convex approximation," IEEE Trans. Signal Processing, vol. 64, no. 11, pp. 2788-2802, Jun. 2016. 